\title{
Left anterior descending coronary artery dissection after blunt chest trauma: Assessment by multi-detector row computed tomography
}

\author{
Tarek Smayra, MD, ${ }^{a}$ Roger Noun, MD, ${ }^{\mathrm{b}}$ and Carla Tohmé-Noun, MD, ${ }^{a}$ Beirut, Lebanon
}

A

17-year-old male patient with no previous heart disease was sent to our institution for multi-detector row computed tomography (MDCT) coronary angiography following posttraumatic myocardial infarction.

\section{Clinical Summary}

Two months earlier, this unrestrained driver had an accident and sustained steering wheel injury as well as head trauma and multiple fractures. He was admitted to the intensive care unit for loss of consciousness at the scene and had his fractures repaired. Follow-up was unremarkable and he was discharged 1 month after the accident. A few days later, he presented with chest pain associated with ST elevation in the precordial leads on electrocardiogram and high serum level of creatine kinase with a positive MB fraction. Transthoracic echocardiography showed moderate hypokinesis of the septum and the anterior wall. MDCT coronary angiography was carried out using a 64-slice LightSpeed VCT (General Electric Healthcare, Little Chalfont, UK) with 0.625-mm collimation, cardiac gating, and intravenous injection of iopromide $370 \mathrm{mg}$ (milligrams of iodine)/mL (Ultravist, Schering AG, Germany). It showed septoapical and anteroseptal subendocardial linear hypodensity (Figure 1) associated with hypokinesis on cine mode, corresponding to infarcted area of the myocardium. Left ventricle ejection fraction was $43 \%$. Coronary artery analysis showed a left dominant distribution with arterial dissection beginning at the ostium of the left anterior descending coronary artery (LAD) and extending $10 \mathrm{~mm}$ distally with a dilated false lumen giving rise to a septal artery and slightly narrowing the true lumen (Figure 2). The patient had a coronary artery bypass, using the left internal thoracic artery to LAD. A year later, he is doing well and living a normal active life.

\section{Discussion}

Coronary artery dissection is a well-known but unusual complication of blunt chest trauma. Ginzburg and colleagues found only 17 cases published over 20 years, probably because many of these patients present with sudden death. ${ }^{1} \mathrm{LAD}$ is the most frequently injured artery $(76 \%)$ followed by the right coronary (12\%) and the

From the Radiology Department ${ }^{\mathrm{a}}$ and Surgery Department, ${ }^{\mathrm{b}}$ Hotel-Dieu hospital, Beirut, Lebanon.

Received for publication Nov 13, 2006; accepted for publication Nov 20, 2006.

Address for reprints: Tarek Smayra, MD, Radiology Department, Hotel-Dieu hospital, Adib Ishak str, 16-6830, Beirut, Lebanon (E-mail: tarek.smayra@ hdf.usj.edu.lb or tarek_smayra@hotmail.com).

J Thorac Cardiovasc Surg 2007;133:811-2

$0022-5223 / \$ 32.00$

Copyright () 2007 by The American Association for Thoracic Surgery

doi:10.1016/j.jtcvs.2006.11.026 circumflex (6\%) arteries. The dissection begins with a small intimal tear caused by the combination of sudden compression of the anterior chest wall and shearing forces following the impact. ${ }^{2}$ Intimal tear evolves under hemodynamic trauma, and disruption of endothelial lining causes coronary thrombosis and myocardial infarction (MI), the most frequent mode of presentation of coronary artery dissection, as in our patient. ${ }^{3}$ In addition to the young age of the patient, the circumstance of this MI, occurring less than 1 month after a violent trauma, must evoke traumatic etiology. Coronary angiography is the established diagnostic procedure to accurately depict intimal tear or thrombosis, but this invasive technique carries a hypothetical risk of worsening a small proximal dissection through catheter manipulation. In some cases, intravascular ultrasound is reliable in the assessment of the dissection and in making good therapeutic choices. ${ }^{3,4}$ However, this special procedure, which requires sedation, is also invasive and rarely available. Nowadays, with the growing use of noninvasive imaging techniques, especially 64-slice scanners, the diagnosis of coronary artery dissection has become very easy. MDCT shows coronary artery dissection in the classic form of a dilated artery with 2 parallel lumens, separated by the intimal flap. The true lumen is slightly narrowed by a larger, aneurysmal false one, as in our patient. A complete or partial thrombosis of the false lumen is

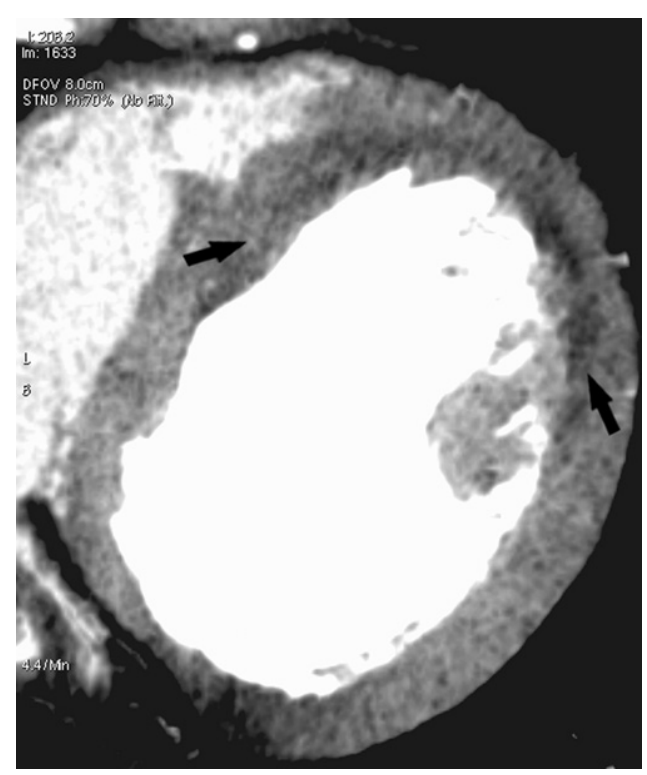

Figure 1. Enhanced MDCT axial image showing subendocardial linear hypodensity (arrows) of the anteroseptal and anterolateral myocardium of the left ventricle, corresponding to poorly enhanced, infarcted area. MDCT, multi-detector row computed tomography. 


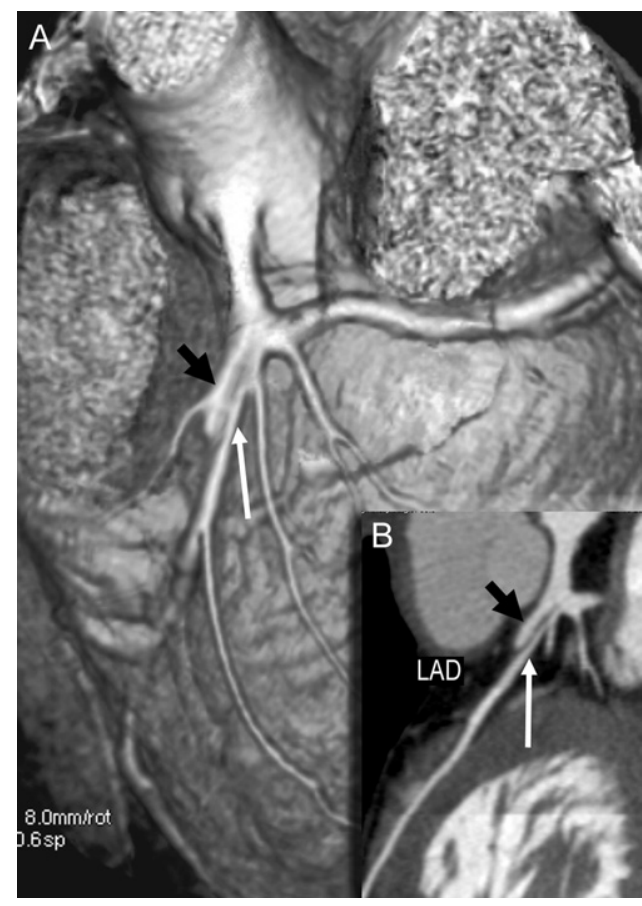

Figure 2. Volume rendering (A) and curvilinear (B) analysis of left anterior descending artery (LAD) showing intimal tear at the ostium with a dilated false lumen (black arrow) narrowing the true lumen (white arrow).

possible in the absence of a reentry point. MDCT allows a complete, noninvasive management workup with a thorough analysis of the dissected segment and its relationship with different coronary branches as well as associated myocardial anomalies, posttraumatic valvular apparatus disruption, and other thoracic inju- ries. Drawbacks of MDCT are the use of ionizing radiations, iodinated contrast material, and the need for regular and slow pulse rate in a conscious and cooperative patient. Some authors advocate conservative management of posttraumatic coronary artery dissection, relying on spontaneous healing of intimal tear. ${ }^{2,3}$ The use of thrombolytic agents may worsen the dissection itself, not to mention the high risk of bleeding from other posttraumatic lesions, and is not systematically recommended. Revascularization through coronary artery bypass is the most frequent treatment found in the literature, especially in the presence of other cardiac lesions, such as valvular disruption, requiring simultaneous surgical repair. ${ }^{5}$ Endovascular stenting has been performed many times with good long-term results. ${ }^{1,3}$ With the expected increase of use of MDCT in trauma patients, more coronary artery dissections will be diagnosed, raising the question of whether to stent these asymptomatic patients.

This unique case shows the importance of MDCT as a first-line noninvasive diagnostic tool in suspected posttraumatic coronary lesions.

\section{References}

1. Ginzburg E, Dygert J, Parra-Davila E, Lynn M, Almeida J, Mayor M. Coronary artery stenting for occlusive dissection after blunt chest trauma. J Trauma. 1998;45:157-61.

2. Yoon SJ, Kwon HM, Kim DS, Hong BK, Kim DY, Cho YH, et al Acute myocardial infarction caused by coronary artery dissection following blunt chest trauma. Yonsei Med J. 2003;44:736-9.

3. Chun JH, Lee SC, Gwon HC, Lee SH, Hong KP, Seo JD, et al. Left main coronary artery dissection after blunt chest trauma presented as acute anterior myocardial infarction: assessment by intravascular ultrasound: a case report. J Korean Med Sci. 1998;13:325-7.

4. Moreno R, Perez del Todo J, Nieto M, Alba F, Alfonso F, GarciaRubira JC, et al. Primary stenting in acute myocardial infarction secondary to right coronary artery dissection following blunt chest trauma. Usefulness of intracoronary ultrasound. Int J Cardiol. 2005; 103:209-11.

5. Reiss J, Razzouk AJ, Kiev J, Bansal R, Bailey LL. Concomitant traumatic coronary artery and tricuspid valve injury: a heterogeneous presentation. J Trauma. 2001;50:942-4. 